\title{
RACSAM
}

Rev. R. Acad. Cien. Serie A. Mat.

VOL. 102 (2), 2008, pp. 237-249

Ciencias de la Computación / Computational Sciences

\section{An Axiom System for Incidence Spatial Geometry}

\author{
R. Rubio and A. Ríder
}

\begin{abstract}
Incidence spatial geometry is based on three-sorted structures consisting of points, lines and planes together with three intersort binary relations between points and lines, lines and planes and points and planes. We introduce an equivalent one-sorted geometrical structure, called incidence spatial frame, which is suitable for modal considerations. We are going to prove completeness by SD-Theorem. Extensions to projective, affine and hyperbolic geometries are also considered.
\end{abstract}

\section{Un sistema axiomático para la geometría espacial de incidencia}

Resumen. La geometría espacial de incidencia está construida por medio de estructuras trisurtidas formadas por puntos, rectas y planos con relaciones binarias de interconexión, para cada dos de estos elementos. En este trabajo introducimos una estructura monosurtida, que denominamos marco espacial de incidencia y que resulta adecuada para un tratamiento modal. Probaremos la completitud del sistema por medio del SD-teorema. Las extensiones a los casos proyectivo, afín e hiperbólico son también considerados.

Deductive logic systems are useful tools for knowledge engineering and computer sciences. This development has turned into a master research line in mathematical logic. In particular, the systems based on modal languages are especially adequate for computation sciences. Under this approach, several systems have been studied which model temporal relations in their different interpretations and aspects of use, so that the logic of time is a well-established branch of modal logic, whereas the same cannot be said of the logic of space. The reason is probably in the more simple mathematical structure of time: a set of moments together with a precedence relation between moments [9]. Such one-sorted relational structures are very suitable for a modal treatment. It is quite apparent that incidence spatial relations are more complex than temporal ones. Therefore, these structures are composed of different objects (i.e., they are many-sorted structures): points, lines, planes, etc. Also, these objects are linked by several relations: collinearity, parallelism, orthogonality, incidence, etc. However, the study of geometrical models and their logical theories is justified from an applied perspective since these logical systems can be used to formalize various aspect and modes of practical spatial reasoning, such as reasoning on geographic charts, robotics, etc.

Several authors have been progressively working on geometrical properties and relations from the point of view of these modal languages. Von Wright [19] has introduced a modal logic of space whose modalities were "near" and "somewhere". Van Benthem [5] and Goldblatt [10] independently advocated the use of modal logic to describe relations between events but were not able to propose any intensional model of space. Recently, Balbiani and co-authors have been working on geometrical properties and relations concerning to the plane geometry, from the point of view of new multimodal and many-sorted languages [2, $3,4]$.

Presentado por / Submitted by Luis Laita de la Rica.

Recibido / Received: 20 de noviembre 2007 Aceptado / Accepted: 6 de febrero de 2008 .

Palabras clave / Keywords: Modal Logic, Incidence Spatial Geometry.

Mathematics Subject Classifications: 03B45, 03B70, 68Q17

(C) 2008 Real Academia de Ciencias, España. 
In this work, following [16, 14, 15], we build a one-sorted structure (with only one class of objects) which will be adequate for classical modal considerations and able to capture in an equivalent way the models of incidence spatial geometry (dimension three) and we build a classical axiomatic multimodal system suitable to be valuated on these one-sorted structures. This idea of an abstract frame for representing knowledge about different types of data has its origin in the modal theory of arrows developed by Vakarelov [16].

We also prove correctness and completeness for our systems and extensions to affine, projective and hyperbolic geometries are also considered. On the other hand, decidibility is still an open problem.

\section{Incidence Spatial Geometry}

In this section we introduce the incidence spatial geometries and incidence spatial frame, which will be later needed when we assign the semantic to an axiomatic system. As incidence spatial frames are defined by simply encoding the information concerning points, lines and planes in a different way, let us remember the structure of incidence spatial geometry. Finally, we show that these categories are equivalent.

\subsection{The category of incidence spatial geometries}

By an incidence spatial geometry we will mean any three-sorted structure $S=\left(P, L, \Pi, \operatorname{in}_{1}, \operatorname{in}_{2}, \operatorname{in}_{3}\right)$, which satisfies:

1. $P$ is a non-empty set, whose elements are called points, denoted by lower case letters $x, y, z$, etc...,

2. $L$ is a non-empty set, whose elements are called lines, denoted by capital letters $X, Y, Z$, etc...,

3. $\Pi$ is a non-empty set, whose elements are called planes, denoted by lower bold letters $\mathbf{x}, \mathbf{y}, \mathbf{z}$, etc...

4. in $_{1}$ is an intersort relation between points and lines (in $\left.{ }_{1} \subset P \times L\right)$,

5. $\mathrm{in}_{2}$ is an intersort relation between points and planes $\left(\operatorname{in}_{2} \subset P \times \Pi\right)$,

6. in $_{3}$ is an intersort relation between lines and planes $\left(\mathrm{in}_{3} \subset L \times \Pi\right)$.

Moreover we have the following axioms:

I0 $P \cap L=\emptyset, P \cap \Pi=\emptyset, L \cap \Pi=\emptyset$.

I1 $(\forall x, y \in P)(\exists Z \in L)\left(\left(x\right.\right.$ in $\left._{1} Z\right) \wedge\left(y\right.$ in $\left.\left._{1} Z\right)\right)$, two points are together incident with at least one line.

I2 $(\forall x, y \in P)(\forall Z, T \in L)\left(\left(\left(x \operatorname{in}_{1} Z\right) \wedge\left(y \operatorname{in}_{1} Z\right) \wedge\left(x \operatorname{in}_{1} T\right) \wedge\left(y \operatorname{in}_{1} T\right)\right) \rightarrow((x=\right.$ $y) \vee(Z=T)))$, two different points are together incident with at most one line.

I3 $(\forall Z \in L)(\exists x, y \in P)\left((x \neq y) \wedge\left(x \operatorname{in}_{1} Z\right) \wedge\left(y \operatorname{in}_{1} Z\right)\right)$, each line contains at least two different points.

I4 $(\forall x \in P)(\exists Z, T \in L)\left((Z \neq T) \wedge\left(x i n_{1} Z\right) \wedge\left(x\right.\right.$ in $\left.\left._{1} T\right)\right)$, each point belongs to at least two different lines.

I5 $(\forall x \in P)(\forall Y \in L)(\exists \mathbf{z} \in \Pi)\left(\left(x\right.\right.$ in $\left._{2} \mathbf{z}\right) \wedge\left(Y\right.$ in $\left.\left._{3} \mathbf{z}\right)\right)$, one point and one line are together incident with at least one plane.

I6 $(\forall x \in P)(\forall Y \in L)(\forall \mathbf{z}, \mathbf{t} \in \Pi)\left(\left(\left(x \operatorname{in}_{2} \mathbf{z}\right) \wedge\left(Y \operatorname{in}_{3} \mathbf{z}\right) \wedge\left(x \operatorname{in}_{2} \mathbf{t}\right) \wedge\left(Y \operatorname{in}_{3} \mathbf{t}\right)\right) \rightarrow\left(\left(x \operatorname{in}_{1} Y\right) \vee\right.\right.$ $(\mathbf{z}=\mathbf{t})))$, one point and one line, which aren't incident, are incident with at most one plane.

I7 $(\forall \mathbf{z} \in \Pi)(\exists X, Y \in L)\left((X \neq Y) \wedge\left(X \operatorname{in}_{3} \mathbf{z}\right) \wedge\left(Y \operatorname{in}_{3} \mathbf{z}\right)\right)$, each plane contains at least two different lines. 
I8 $(\forall x, y \in P)(\forall Z \in L)(\forall \mathbf{t} \in \Pi)\left(\left(\left(x \operatorname{in}_{1} Z\right) \wedge\left(y \operatorname{in}_{1} Z\right) \wedge\left(x \operatorname{in}_{2} \mathbf{t}\right) \wedge\left(y \operatorname{in}_{2} \mathbf{t}\right)\right) \rightarrow\left(\left(Z \operatorname{in}_{3} \mathbf{t}\right)\right)\right)$, if two points are together incident with one line and one plane, then the line will be incident with the plane.

I9 $(\forall x \in P)(\forall \mathbf{z}, \mathbf{t} \in \Pi)(\exists y, z \in P)\left(\left(\left(x \operatorname{in}_{2} \mathbf{z}\right) \wedge\left(x \operatorname{in}_{2} \mathbf{t}\right)\right) \rightarrow\left((y \neq z) \wedge\left(y \operatorname{in}_{2} \mathbf{z}\right) \wedge\left(y \operatorname{in}_{2} \mathbf{t}\right) \wedge\right.\right.$ $\left.\left.\left(z \operatorname{in}_{2} \mathbf{z}\right) \wedge\left(z \operatorname{in}_{2} \mathbf{t}\right)\right)\right)$, if one point is incident with two planes, then the planes contain at least two common points.

I10 $(\forall X \in L)(\exists \mathbf{y}, \mathbf{z} \in \Pi)\left((\mathbf{z} \neq \mathbf{t}) \wedge\left(X\right.\right.$ in $\left._{3} \mathbf{y}\right) \wedge\left(X\right.$ in $\left.\left._{3} \mathbf{z}\right)\right)$, each line belongs to at least two different planes.

This geometry is known as absolute or neutral geometry and is presented in many textbooks on geometry, for example in $[11,12]$. The class of all incidence spatial geometries is denoted by $\Sigma$. We consider $\Sigma$ as a category where the objects are the incidence spatial geometries and the homomorphism are defined as follows: Let $S=\left(P, L, \Pi, \mathrm{in}_{1}, \mathrm{in}_{2}, \mathrm{in}_{3}\right)$ and $S^{\prime}=\left(P^{\prime}, L^{\prime}, \Pi^{\prime}, \mathrm{in}_{1}^{\prime}, \mathrm{in}_{2}^{\prime}, \mathrm{in}_{3}^{\prime}\right)$ be incidence spatial geometries. A map $f$ from $P \cup L \cup \Pi$ onto $P^{\prime} \cup L^{\prime} \cup \Pi^{\prime}$ is called a homomorphism from $S$ into $S^{\prime}$ if:

- for any $(x, Y, \mathbf{z}) \in S$ we have $(f(x), f(Y), f(\mathbf{z})) \in S^{\prime}$,

- for any $(x, Y, \mathbf{z}) \in S$,

- if $x \operatorname{in}_{1} Y$ then $f(x) \operatorname{in}_{1}^{\prime} f(Y)$,

- if $x \operatorname{in}_{2} \mathbf{z}$ then $f(x) \operatorname{in}_{2}^{\prime} f(\mathbf{z})$,

- if $Y \operatorname{in}_{3} \mathbf{z}$ then $f(Y) \operatorname{in}_{3}^{\prime} f(\mathbf{z})$.

An homomorphism $f$ which admits an inverse homomorphism is called an isomorphism.

Incidence spatial geometries are in a sense minimal geometrical structures, which can be extended with new axioms. Three natural extensions are projective, affine and hyperbolic geometries.

An incidence spatial geometry $S=\left(P, L, \Pi, \mathrm{in}_{1}, \mathrm{in}_{2}, \mathrm{in}_{3}\right)$ is called projective if it satisfies the following additional axioms:

P1 $(\forall Z, T \in L)(\forall \mathbf{z} \in \Pi)(\exists x \in P)\left(\left(\left(Z\right.\right.\right.$ in $\left._{3} \mathbf{z}\right) \wedge\left(T\right.$ in $\left.\left._{3} \mathbf{z}\right)\right) \rightarrow\left(\left(x\right.\right.$ in $\left._{1} Z\right) \wedge\left(x\right.$ in $\left.\left.\left._{1} T\right)\right)\right)$, two lines (on a plane) contain at least one point in common.

P2 $(\forall T \in L)(\forall x, y \in P)(\exists z \in P)\left(\left(\left(x \operatorname{in}_{1} Z\right) \wedge\left(y \operatorname{in}_{1} Z\right)\right) \rightarrow\left(\left(z \operatorname{in}_{1} T\right) \wedge(z \neq x) \wedge(z \neq y)\right)\right)$, if a line contains two points $x$ and $y$ then it contains at least one point $z$ different from $x$ and $y$.

These axioms are presented in [7].

To introduce the notion of affine spatial geometry we first define the relation of parallelism between lines. Two lines $Z$ and $T$ that lie in a plane are parallel $(Z \| T)$ if $Z=T$ or they do not intersect. We denote parallelism by $Z \| T$.

$$
Z \| T \quad \text { iff } \quad(\exists \mathbf{z} \in \Pi)\left(Z \operatorname{in}_{3} \mathbf{z} \wedge T \operatorname{in}_{3} \mathbf{z}\right) \wedge(\forall x \in P)\left(x \operatorname{in}_{1} Z \wedge x \operatorname{in}_{1} T \rightarrow Z=T\right) .
$$

An incidence spatial geometry $S=\left(P, L, \Pi, \mathrm{in}_{1}, \mathrm{in}_{2}, \mathrm{in}_{3}\right)$ is called affine if it satisfies the following additional axioms:

A1 $(\forall x \in P)(\forall Y \in L)(\exists Z \in L)\left(\left((Y \| Z) \wedge\left(x \operatorname{in}_{1} Z\right)\right) \vee\left(x \operatorname{in}_{1} Y\right)\right)$, for every line $Y$ and every point $x$ that does not lie on $Y$ there is at least one line $Z$ through $x$ that is parallel to $Y$.

A2 $(\forall X, Y, Z \in L)(((X \| Y) \wedge(Y \| Z)) \rightarrow((X \| Z)))$, the relation $\|$ is transitive. 
From these axioms and the definition of $\|$, we can prove that there is at most one line both incident with a given point and parallel to a given line. Thus, this axiomatic is equivalent to that considered by Hilbert [12].

An incidence spatial geometry $S=\left(P, L, \Pi, \mathrm{in}_{1}, \mathrm{in}_{2}, \mathrm{in}_{3}\right)$ is called hyperbolic if it satisfies the following additional axiom:

H1 $(\forall x \in P)(\forall Y \in L)(\exists Z, T \in L)\left((Z \neq T) \wedge(Y \| Z) \wedge(Y \| T) \wedge\left(x \operatorname{in}_{1} Z\right) \wedge\left(x \operatorname{in}_{1} T\right)\right) \vee$ $\left(x \operatorname{in}_{1} Y\right)$ ), for every line $Y$ and every point $x$ not on $Y$ there exists at least two lines through $x$ parallel to $Y$.

This axiomatic are presented in [11].

\subsection{The category of incidence spatial frames}

The notion of incidence spatial geometry as a three-sorted relational structure with three intersort binary relations is not suitable as a semantical basis of some modal logic. That is why we introduce new structures, called incidence spatial frames, which are one-sorted and can be used for modal semantics.

Let $S=\left(P, L, \Pi, \mathrm{in}_{1}, \mathrm{in}_{2}, \mathrm{in}_{3}\right)$ be an incidence spatial geometry. The relational structure $W(S)=$ $\left(W, \equiv_{1}, \equiv_{2}, \equiv_{3}\right)$ is called an incidence spatial frame over $S$ if the following conditions are satisfied:

1. $W=\left\{(x, Y, \mathbf{z}): x \in P, Y \in L, \mathbf{z} \in \Pi, x \operatorname{in}_{1} Y, x \operatorname{in}_{2} \mathbf{z}, Y\right.$ in $\left._{3} \mathbf{z}\right\}$,

2. $\left(x_{1}, Y_{1}, \mathbf{z}_{1}\right) \equiv_{1}\left(x_{2}, Y_{2}, \mathbf{z}_{2}\right)$ iff $x_{1}=x_{2}$,

3. $\left(x_{1}, Y_{1}, \mathbf{z}_{\mathbf{1}}\right) \equiv_{2}\left(x_{2}, Y_{2}, \mathbf{z}_{\mathbf{2}}\right)$ iff $Y_{1}=Y_{2}$,

4. $\left(x_{1}, Y_{1}, \mathbf{z}_{\mathbf{1}}\right) \equiv_{3}\left(x_{2}, Y_{2}, \mathbf{z}_{\mathbf{2}}\right)$ iff $\mathbf{z}_{\mathbf{1}}=\mathbf{z}_{\mathbf{2}}$.

The element $t=(x, Y, \mathbf{z})$ of $W$ can be considered as point, line or plane according to the context in which $t$ occurs. For instance in the expression $t \equiv_{1} v$, the elements $t$ and $v$ are meant as points and the relation $\equiv_{1}$ can be understood as the equality of points. In the structure $W(S)$ we can define three relations $\mathrm{on}_{1}, \mathrm{on}_{2}$ and $\mathrm{on}_{3}$ in the following way:

$$
\begin{array}{lll}
\left(x_{1}, Y_{1}, \mathbf{z}_{\mathbf{1}}\right) \mathrm{on}_{1}\left(x_{2}, Y_{2}, \mathbf{z}_{\mathbf{2}}\right) & \text { iff } & x_{1} \mathrm{in}_{1} Y_{2}, \\
\left(x_{1}, Y_{1}, \mathbf{z}_{\mathbf{1}}\right) \mathrm{on}_{2}\left(x_{2}, Y_{2}, \mathbf{z}_{\mathbf{2}}\right) & \text { iff } & x_{1} \mathrm{in}_{2} \mathbf{z}_{\mathbf{2}}, \\
\left(x_{1}, Y_{1}, \mathbf{z}_{\mathbf{1}}\right) \mathrm{on}_{3}\left(x_{2}, Y_{2}, \mathbf{z}_{\mathbf{2}}\right) & \text { iff } & Y_{1} \mathrm{in}_{3} \mathbf{z}_{\mathbf{2}} .
\end{array}
$$

The relations converse of $\mathrm{on}_{i}$ is denoted by on ${ }_{i}^{-1}$ for $i \in\{1,2,3\}$.

The following shows that $\mathrm{on}_{i}$ and $\mathrm{on}_{i}^{-1}$, for $i=\{1,2,3\}$ are definable as compositions of the three relations $\equiv_{1}, \equiv_{2}$ and $\equiv_{3}$.

Lemma 1 Let $S$ be one incidence spatial geometry and $W(S)$ be the incidence spatial frame over $S$. Then for any $x, y \in W$, we have:

(i) $x$ on $_{1} y$ iff $(\exists z \in W)\left(\left(x \equiv_{1} z\right) \wedge\left(z \equiv_{2} y\right)\right)$ iff $\left(\mathrm{on}_{1}=\equiv_{1} \circ \equiv_{2}\right)$,

(ii) $x \mathrm{on}_{2} y$ iff $(\exists z \in W)\left(\left(x \equiv_{1} z\right) \wedge\left(z \equiv_{3} y\right)\right)$ iff $\left(\mathrm{on}_{2}=\equiv_{1} \circ \equiv_{3}\right)$,

(iii) $x$ on $_{3} y$ iff $(\exists z \in W)\left(\left(x \equiv_{2} z\right) \wedge\left(z \equiv_{3} y\right)\right)$ iff $\left(\mathrm{on}_{3}=\equiv_{2} \circ \equiv_{3}\right)$,

(iv) $x \mathrm{on}_{1}^{-1} y$ iff $(\exists z \in W)\left(\left(x \equiv_{2} z\right) \wedge\left(z \equiv_{1} y\right)\right)$ iff $\left(\mathrm{on}_{1}^{-1}=\equiv_{2} \circ \equiv_{1}\right)$,

(v) $x \mathrm{on}_{2}^{-1} y$ iff $(\exists z \in W)\left(\left(x \equiv_{3} z\right) \wedge\left(z \equiv_{1} y\right)\right)$ iff $\left(\mathrm{on}_{1}^{-1}=\equiv_{3} \circ \equiv_{1}\right)$,

(vi) $x \mathrm{on}_{3}^{-1} y$ iff $(\exists z \in W)\left(\left(x \equiv_{3} z\right) \wedge\left(z \equiv_{2} y\right)\right)$ iff $\left(\mathrm{on}_{3}^{-1}=\equiv_{3} \circ \equiv_{2}\right)$. 
PROOF. Straightforward.

Lemma 2 Let $S$ be an incidence spatial geometry and $W(S)$ be the incidence spatial frame over $S$. Then the following conditions are satisfied:

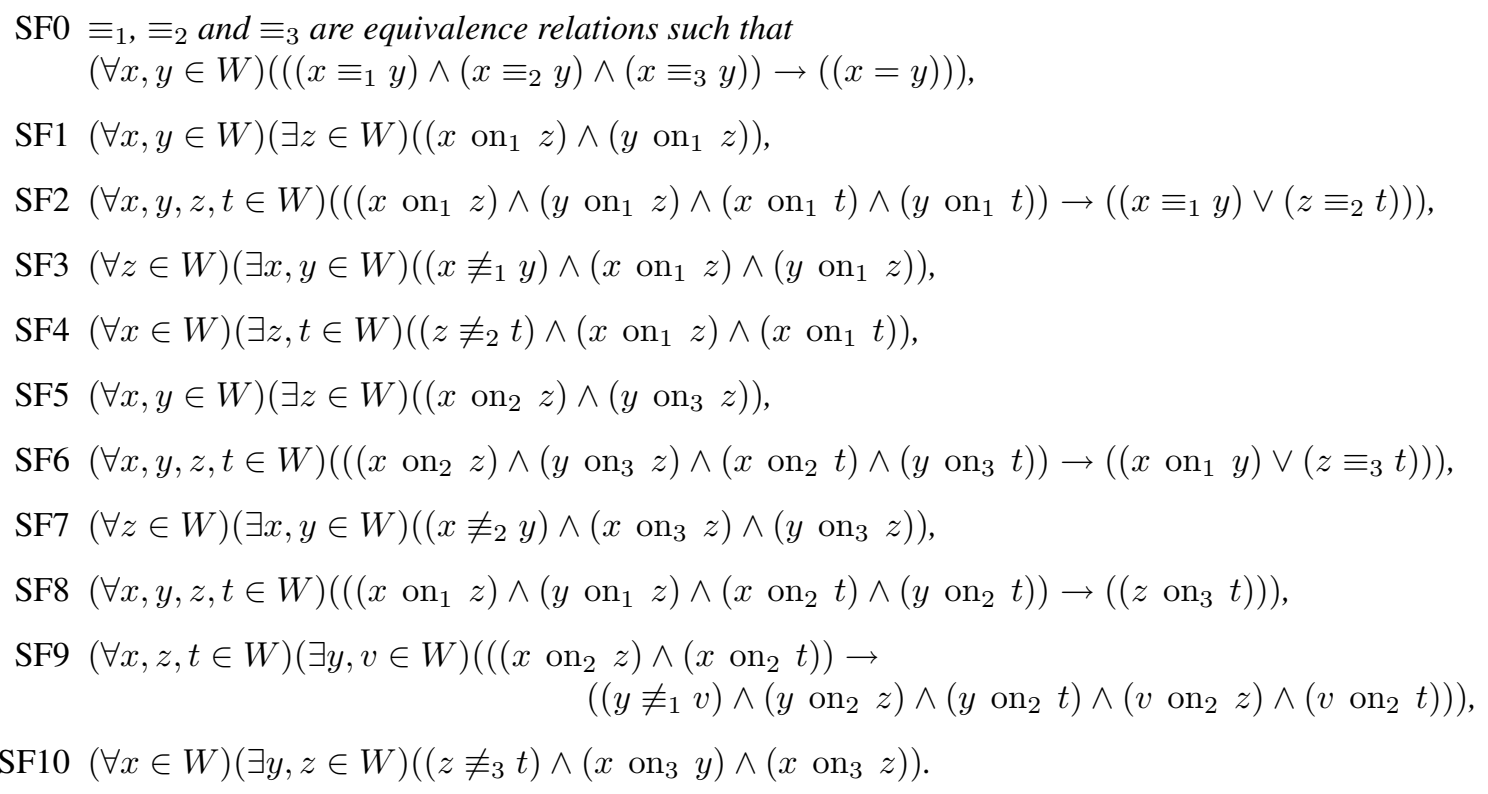

If $S$ be an incidence spatial projective geometry then we have:

SPF1 $(\forall y, z, t \in W)(\exists x \in W)\left(\left(\left(y\right.\right.\right.$ on $\left._{3} t\right) \wedge\left(z\right.$ on $\left.\left._{3} t\right)\right) \rightarrow\left(\left(x\right.\right.$ on $\left._{1} y\right) \wedge\left(x\right.$ on $\left.\left.\left._{1} z\right)\right)\right)$,

SPF2 $(\forall x, y, z \in W)(\exists t \in W)\left(\left(\left(x\right.\right.\right.$ on $\left._{1} z\right) \wedge\left(y\right.$ on $\left.\left._{1} z\right)\right) \rightarrow\left(\left(t\right.\right.$ on $\left.\left.\left._{1} z\right) \wedge(t \neq x) \wedge(t \neq y)\right)\right)$.

If $S$ be an incidence spatial affine geometry then we have:

SAF1 $(\forall x, y \in W)(\exists z \in W)\left((y \| z) \wedge\left(x\right.\right.$ on $\left._{1} z\right) \vee\left(x\right.$ on $\left.\left._{1} y\right)\right)$,

$\mathrm{SAF} 2(\forall x, y, z \in W)(((x \| y) \wedge(y \| z)) \rightarrow((x \| z)))$.

If $S$ be an incidence spatial hyperbolic geometry then we have:

SHF1 $(\forall x, y \in W)(\exists z, t \in W)\left((z \neq t) \wedge(y \| z) \wedge(y \| t) \wedge\left(x\right.\right.$ on $\left._{1} z\right) \wedge\left(x\right.$ on $\left.\left._{1} t\right) \vee\left(\begin{array}{lll}x & \text { on }_{1} & y\end{array}\right)\right)$.

PROOF. Straightforward.

We define incidence spatial frame as any relational structure $W_{f}=\left(W, \equiv_{1}, \equiv_{2}, \equiv_{3}\right)$ with $W \neq \emptyset$ and satisfying the axioms SF0...SF10 from lemma 2. By an incidence spatial projective frame we will mean any incidence spatial frame, satisfying the axioms SPF1 and SPF2 from lemma 2. We define incidence spatial affine frame as any incidence spatial frame, satisfying the axioms SAF1 and SAF2 from lemma 2. In the same way we define incidence spatial hyperbolic frame as any incidence spatial frame, satisfying the axiom SHF1 from lemma 2. The class of all incidence spatial frames is denoted by $\Phi$. We consider $\Phi$ as a category where morphisms are the usual homomorphisms between one-sorted relational structure. If $W_{f}=\left(W, \equiv_{1}, \equiv_{2}, \equiv_{3}\right)$ and $W_{f}^{\prime}=\left(W^{\prime}, \equiv_{1}^{\prime}, \equiv_{2}^{\prime}, \equiv_{3}^{\prime}\right)$ are incidence spatial frames, then the map $f$ is called homomorphism from $W_{f}$ to $W_{f}^{\prime}$ if it satisfies: for any $x, y \in W$, if $x \equiv_{i} y$ then $f(x) \equiv_{i}^{\prime} f(y), i=1,2,3$. An homomorphism $f$ from $W_{f}$ onto $W_{f}^{\prime}$ is called isomorphism if it satisfies:

(i) $f$ is a bijective map from $W_{f}$ onto $W_{f}^{\prime}$,

(ii) for any $x, y \in W$, if $f(x) \equiv_{i}^{\prime} f(y)$ then $x \equiv_{i} y, i=1,2,3$. 


\subsection{Equivalence of categories}

In this section we prove categorical equivalence of the categories $\Sigma$ and $\Phi$ of incidence spatial geometries and incidence spatial frames. First we shall give a construction of an incidence spatial geometry from a given incidence spatial frame. Let $W_{f}=\left(W, \equiv_{1}, \equiv_{2}, \equiv_{3}\right)$ be an incidence spatial frame. We define an incidence spatial geometry $S\left(W_{f}\right)$ called the incidence spatial geometry over $W_{f}$, as follows: Let $x \in W$, the equivalence class of $x$ determined by the equivalence relation $\equiv_{i}$ will be denoted by $|x|_{i}, i=1,2,3$.

$$
\begin{gathered}
P_{S}\left(W_{f}\right)=P=W / \equiv_{1}=\left\{|x|_{1}: x \in W\right\}, \\
L_{S}\left(W_{f}\right)=L=W / \equiv_{2}=\left\{|x|_{2}: x \in W\right\}, \\
\Pi_{S}\left(W_{f}\right)=\Pi=W / \equiv_{3}=\left\{|x|_{3}: x \in W\right\}, \\
\mathrm{in}_{1 S}=\operatorname{in}_{1}=\left\{\left(|x|_{1},|y|_{2}\right): x, y \in W, x \text { on }_{1} y\right\}, \\
\operatorname{in}_{2 S}=\operatorname{in}_{2}=\left\{\left(|x|_{1},|y|_{3}\right): x, y \in W, x \text { on }_{2} y\right\}, \\
\operatorname{in}_{3 S}=\operatorname{in}_{3}=\left\{\left(|x|_{2},|y|_{3}\right): x, y \in W, x \text { on }_{3} y\right\} .
\end{gathered}
$$

Lemma 3 Let $W_{f}$ be an incidence spatial frame, then $S\left(W_{f}\right)$ is an incidence spatial geometry.

PROOF. Straightforward.

Theorem 1 If $W_{f}=\left(W, \equiv_{1}, \equiv_{2}, \equiv_{3}\right)$ is an incidence spatial frame, then the frame $W_{f}^{\prime}=W\left(S\left(W_{f}\right)\right)$ is isomorphic with $W_{f}$.

Proof. We define the map $f: W_{f} \rightarrow W_{f}^{\prime}$ as

$$
f(x)=\left(|x|_{1},|x|_{2},|x|_{3}\right), \forall x \in W .
$$

The map $f$ is well defined and it is an isomorphism.

Theorem 2 If $S=\left(P, L, \mathrm{in}_{1}, \mathrm{in}_{2}, \mathrm{in}_{3}\right)$ is an incidence spatial geometry, the incidence spatial geometry $S^{\prime}=S(W(S))$ is isomorphic with $S$.

Proof. We define the map $f: S \rightarrow S^{\prime}$ as

$$
\begin{aligned}
& f(x)=\left|\left(x, Y_{1}, \mathbf{z}_{\mathbf{1}}\right)\right|_{1}, \quad \forall x \in P, \\
& f(Y)=\left|\left(x_{2}, Y, \mathbf{z}_{2}\right)\right|_{2}, \quad \forall Y \in L, \\
& f(\mathbf{z})=\left|\left(x_{3}, Y_{3}, \mathbf{z}\right)\right|_{3}, \quad \forall \mathbf{z} \in \Pi .
\end{aligned}
$$

where, for instance, the existence of $Y_{1}, \mathbf{z}_{1}$ are given by the axioms (I4) and (I5).

The map $f$ is well defined and it is an isomorphism.

Consequently $W$ and $S$ are maps between objects of both categories $\Sigma$ and $\Phi$ :

$$
\begin{gathered}
W: \Sigma \rightarrow \Phi, \\
S: \Phi \rightarrow \Sigma .
\end{gathered}
$$

Let $f$ be a homomorphism between two incidence spatial geometries $S$ and $S^{\prime}$. We define the mapping:

$$
W(f): W(S) \mapsto W\left(S^{\prime}\right)
$$

as follows:

$$
\text { If }(x, Y, \mathbf{z}) \in W \text {, then } W(f)((x, Y, \mathbf{z}))=(f(x), f(Y), f(\mathbf{z})) \text {. }
$$


This definition makes commutative the following diagram and it converts $W$ in a functor from $\Sigma$ into $\Phi$.

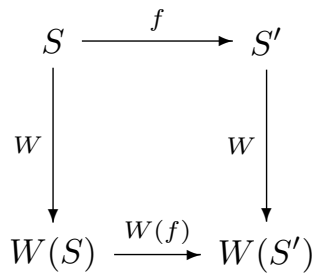

Analogously, if $f: W \mapsto W^{\prime}$ is a homomorphism of incidence spatial frames, the mapping:

$$
S(f): S\left(W_{f}\right) \mapsto S\left(W_{f}^{\prime}\right),
$$

defined as:

$$
\left.S(f)\left(|x|_{1}\right)=|f(x)|_{1}, \quad S(f)\left(|Y|_{2}\right)=|f(Y)|_{2}, \quad S(f)\left(|\mathbf{z}|_{3}\right)=|f(\mathbf{z})|_{3}\right),
$$

makes commutative the following diagram and it converts $S$ into a functor from $\Phi$ into $\Sigma$.

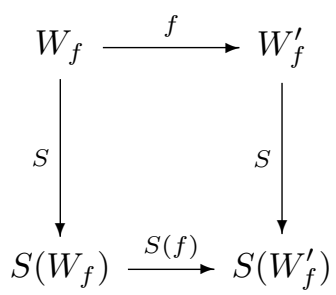

To conclude this section we refer the reader to the well-known theorem about categories ([13, proposition 1.3]), which proves the equivalence of categories.

\section{The modal logic of incidence spatial geometry}

The class of incidence spatial frames, denoted by MISG is a suitable reference for the kripke semantic construction (or relational semantics, that introduces many explicit accessibility relations) for a modal logic of incidence spatial geometry. We begin introducing the language.

\subsection{Language}

We use multimodal languages with:

1. a set of proposition letters $p, q, r \ldots$,

2. two propositional constants $\top$, true and $\perp$, false,

3. the usual connectives $\neg, \wedge, \vee, \rightarrow, \leftrightarrow$,

4. parentheses $($,$) ,$

5. six unary modal operators $\left[\equiv_{1}\right],\left[\equiv_{2}\right],\left[\equiv_{3}\right],\left[\equiv_{1}\right],\left[\not \equiv_{2}\right],\left[\equiv_{3}\right]$.

The set of well-formed formulas may be defined as usual. We use $A, B, C \ldots$, to denote multimodal formulas. Moreover, we shall often omit parentheses if they are not essential in order to avoid ambiguity, e.g. the outermost parentheses surrounding conjunctions, disjunctions, etc. Thus, we may write $A \wedge B$ for $(A \wedge B)$. In the case of iterated conjunctions or disjunctions we shall agree to associate to the left, e.g. $A \wedge B \wedge C$ will be $((A \wedge B) \wedge C)$. Furthermore, $\wedge$ and $\vee$ shall bind more strongly than $\rightarrow$. Thus $(A \wedge B \rightarrow C)$ will stand for $((A \wedge B) \rightarrow C)$. 


\subsection{Semantics}

The general semantics of the introduced language will be the Kripke semantics over frames of the form $W_{f}=\left(W, \equiv_{1}, \equiv_{2}, \equiv_{3}, \equiv_{1},{\equiv_{2}}_{2}, \equiv_{3}\right)$ where $W \neq \emptyset$ and $\equiv_{1}, \equiv_{2}, \equiv_{3}, \equiv_{1}, \neq_{2}, \neq_{3}$ are arbitrary binary relations in $W$. We shall use several classes of frames and the standard semantics will be the class of all incidence spatial frames, where $\not_{1}, \equiv_{2}, \neq_{3}$ are complement of $\equiv_{1}, \equiv_{2}$ and $\equiv_{3}$, respectively. A valuation $v$ of a frame $W_{f}$ is a function which assigns to each propositional letter $p$ a subset $v(p) \subset W, v(\top)=W$ and $v(\perp)=\emptyset$. The pair $M=\left(W_{f}, v\right)$ is called a model over $W_{f}$. Suppose $x \in W$, then we inductively define the notion of a formula $A$ being satisfied or true in a model $M=\left(W_{f}, v\right)$ at the state $x$ as follows:

$$
\begin{gathered}
x \models_{v} p \text { iff } x \in v(p), \text { where } \mathrm{p} \text { is a propositional letter, } \\
x \models_{v} \neg A \text { iff not } x \models_{v} A, \\
x \models_{v}(A \wedge B) \text { iff } x \models_{v} A \text { and } x \models_{v} B, \\
x \models_{v}(A \vee B) \text { iff } x \models_{v} A \text { or } x \models_{v} B, \\
x \models_{v}(A \rightarrow B) \text { iff } x \models_{v}(\neg A \vee B), \\
x \models_{v}(A \leftrightarrow B) \text { iff } x \models_{v}(A \rightarrow B) \text { and } x \models_{v}(B \rightarrow A), \\
x \models_{v}[R] A \text { iff }(\forall y \in W)\left(x R y \Rightarrow y \models_{v} A\right), \quad R \in\left\{\equiv_{1}, \equiv_{2}, \equiv_{3}, \not \equiv_{1}, \not_{2}, \not \equiv_{3}\right\} .
\end{gathered}
$$

We say that a formula $A$ is true in a frame $W_{f}$ if for any valuation $v$ and any $x \in W$ we have $x \models_{v} A$. We say that $A$ is true in a class of frames $\mathbf{F}$ if $A$ is true in any frame of $\mathbf{F}$. We define the logic of $\mathbf{F}$ as the set of all true formulas in $\mathbf{F}$. The logic of the class of all incidence spatial frames will be denoted by MISG.

We introduce as abbreviations the following definable modal operators:

$$
\begin{gathered}
{[\neq] A=\left[\equiv_{1}\right] A \wedge\left[\not_{2}\right] A \wedge\left[\not_{3}\right] A, \quad[U] A=A \wedge[\neq] A, \quad O A=A \wedge[\neq] \neg A,} \\
{\left[\mathrm{on}_{1}\right] A=\left[\equiv_{1}\right]\left[\equiv_{2}\right] A, \quad\left[\mathrm{on}_{2}\right] A=\left[\equiv_{1}\right]\left[\equiv_{3}\right] A, \quad\left[\mathrm{on}_{3}\right] A=\left[\equiv_{2}\right]\left[\equiv_{3}\right] A,} \\
{\left[\mathrm{on}_{1}^{-1}\right] A=\left[\equiv_{2}\right]\left[\equiv_{1}\right] A, \quad\left[\mathrm{on}_{2}^{-1}\right] A=\left[\equiv_{3}\right]\left[\equiv_{1}\right] A, \quad\left[\mathrm{on}_{3}^{-1}\right] A=\left[\equiv_{3}\right]\left[\equiv_{2}\right] A .}
\end{gathered}
$$

If $[R]$ is a modal box then we define the diamond $\langle R\rangle$ as $\neg[R] \neg$.

Must be taken into account, that the negative modalites $\left[\not_{i}\right], i=1,2,3$, are not definable from the positive ones, because they have the irreflexive property (see below).

\section{Axiomatic}

In this section we propose an axiomatic system of MISG.

When we are saying that a modal formula $A$ characterizes a class $K$ of frames, use usually mean that any modal frame $F(W, R)$ is in $K$ if and only if $A$ is valid in $F$ (i.e. in every model $(F, V)$ based on $F, A$ is true in every world of $F$ ). The standard examples are $p \rightarrow\langle R\rangle p$ and $\langle R\rangle\langle R\rangle p \rightarrow\langle R\rangle p$ characterizing respectively the reflexive the transitive frames.

It is also well-known that not all first order definable properties have modal correspondents: the standard example is irreflexivity.

However, these are different ways of characterizing frame classes: consider $\mathrm{d} w$ in a frame $F=(W, R)$. Clearly $w$ is irreflexive if and only if $w \cap\{v / w R v\}=\emptyset$, that is equivalent to saying that we can make the formula $p \rightarrow\langle R\rangle p$ false at $w$. This gives a way of characterizing the irreflexive frame:

$$
F \models \forall x \neg x R x \Leftrightarrow \forall w \exists V\left(F, w \models_{V} \neg(p \rightarrow\langle R\rangle p)\right) .
$$

A different road was taken by Gabbay in [8]. Instead of using axioms, he suggested to add a special derivation rule, which he called irreflexivity rule. This rule can be formulated as follows:

$$
\vdash \neg(p \rightarrow\langle R\rangle p) \rightarrow \phi \Rightarrow \vdash A \text {, if } p \text { is not in } A .
$$


Gabbays's completeness proof then consists of constructing a transitive irreflexive model right away, without passing models that may be bad in the sense that they have reflexive points.

Note that

$$
\neg(p \rightarrow\langle R\rangle p) \leftrightarrow \neg(\neg p \vee\langle R\rangle p) \leftrightarrow(p \wedge \neg\langle R\rangle p) \leftrightarrow(p \wedge[R] \neg(\neg p)) \leftrightarrow(p \wedge[R] \neg p)
$$

is a tautology, so if we define $(p \wedge[R] \neg p)=O p$, then the irreflexivity rule will be:

$$
\vdash O p \rightarrow A \Rightarrow \vdash A \text {, if } p \text { is not in } A \text {. }
$$

We propose the following axiomatization of MISG:

* All tautologies of propositional logic,

$(\mathrm{K})[R](p \rightarrow q) \rightarrow([R] p \rightarrow[R] q), R \in\left\{\equiv_{1}, \equiv_{2}, \equiv_{3}, \neq_{1}, \neq_{2}, \neq_{3}\right\}$,

$$
(\neq \text {-symmetry) }\langle\neq\rangle[\neq] p \rightarrow p,
$$

(f-pseudo-transitivity) $p \wedge[\neq] p \rightarrow[\neq][\neq] p$,

$$
\begin{aligned}
& \text { (f-inclusion) }[U] p \rightarrow[R] p, R \in\left\{\equiv_{1}, \equiv_{2} \equiv_{3}\right\} \text {, } \\
& \mathrm{MISG}^{*}\left[\equiv_{i}\right] p \wedge\left[\not_{i}\right] p \rightarrow[U] p, i=1,2,3, \\
& \mathrm{MISG}^{* *}\left\langle\equiv_{i}\right\rangle[\neq] p \rightarrow[\not \equiv i] p, i=1,2,3, \\
& \text { MISG0 }\left[\equiv_{i}\right] p \rightarrow p,\left\langle\equiv_{i}\right\rangle\left[\equiv_{i}\right] p \rightarrow p,\left[\equiv_{i}\right] p \rightarrow\left[\equiv_{i}\right]\left[\equiv_{i}\right] p, i=1,2,3 \\
& \text { MISG1 }\langle U\rangle p \rightarrow\left\langle\mathrm{on}_{1}\right\rangle\left\langle\mathrm{on}_{1}^{-1}\right\rangle p, \\
& \text { MISG2 }\left\langle\mathrm{on}_{1}\right\rangle\left(p \wedge\left\langle\mathrm{on}_{1}^{-1}\right\rangle([\neq] q \wedge r)\right) \rightarrow\left(\left[\mathrm{on}_{1}\right]\left(\left\langle\equiv_{2}\right\rangle p \vee\left[\mathrm{on}_{1}^{-1}\right] q\right) \vee\left\langle\equiv_{1}\right\rangle r\right), \\
& \text { MISG3 } p \rightarrow\left\langle\mathrm{on}_{1}^{-1}\right\rangle\left\langle\not \equiv{ }_{1}\right\rangle\left\langle\mathrm{on}_{1}\right\rangle p \\
& \text { MISG4 } p \rightarrow\left\langle\mathrm{on}_{1}\right\rangle\left\langle\not_{2}\right\rangle\left\langle\mathrm{on}_{1}^{-1}\right\rangle p \\
& \text { MISG5 }\langle U\rangle p \rightarrow\left\langle\mathrm{on}_{2}\right\rangle\left\langle\mathrm{on}_{3}^{-1}\right\rangle p, \\
& \text { MISG6 }\left\langle\mathrm{on}_{2}\right\rangle\left(p \wedge\left\langle\mathrm{on}_{3}^{-1}\right\rangle([\neq] q \wedge r)\right) \rightarrow\left(\left[\mathrm{on}_{2}\right]\left(\left\langle\equiv_{3}\right\rangle p \vee\left[\mathrm{on}_{3}^{-1}\right] q\right) \vee\left\langle\mathrm{on}_{1}\right\rangle r\right), \\
& \text { MISG7 } p \rightarrow\left\langle\mathrm{on}_{3}^{-1}\right\rangle\langle\not \equiv 2\rangle\left\langle\mathrm{on}_{3}\right\rangle p \\
& \text { MISG8 }\left\langle\mathrm{on}_{1}\right\rangle\left(p \wedge\left\langle\mathrm{on}_{1}^{-1}\right\rangle[\neq] q\right) \rightarrow\left[\mathrm{on}_{2}\right]\left(\left\langle\mathrm{on}_{3}^{-1}\right\rangle p \vee\left[\mathrm{on}_{2}^{-1}\right] q\right), \\
& \text { MISG9 }\left\langle\mathrm{on}_{2}^{-1}\right\rangle\left\langle\mathrm{on}_{2}\right\rangle p \wedge q \rightarrow\left\langle\mathrm{on}_{2}^{-1}\right\rangle\left(\left\langle\equiv_{1}\right\rangle\left(\left\langle\mathrm{on}_{2}\right\rangle p \wedge\left\langle\mathrm{on}_{2}\right\rangle q\right) \wedge\left\langle\mathrm{on}_{2}\right\rangle p\right) \text {, } \\
& \text { MISG10 } p \rightarrow\left\langle\mathrm{on}_{3}\right\rangle\left\langle\not \equiv_{3}\right\rangle\left\langle\mathrm{on}_{3}^{-1}\right\rangle p \text {. }
\end{aligned}
$$

\section{The rules of inference:}

(MP) Modus ponens: if $\vdash A$ and $\vdash A \rightarrow B$ then $\vdash B$,

(NR) Necessitation rule: if $\vdash A$ then $\vdash[R] A, R \in\left\{\equiv_{1}, \equiv_{2}, \equiv_{3}, \equiv_{1}, \neq_{2}, \equiv_{3}\right\}$,

(IR) Irreflexivity rule: if $p$ is not in $A$ and $\vdash O p \rightarrow A$ then $\vdash A$,

(SR) Substitution rule of propositional letters. 


\section{Correctness of MISG}

Here we use Sahlqvist Correspondence Theorem [18] for proving correctness. Next we remember some definitions. An occurrence of a proposition letter $p$ in a formula $A$ (here we must be think $A$ in terms of the conectives $\wedge$ and $\vee$ ) is positive occurrence if it is in the scope of an even number of negations signs; it is a negative occurrence if it is in the scope of an odd number of negations signs. For example, the occurrence of $p$ in $\langle R\rangle(p \rightarrow q)$ is negative, for this formula is shorthand for $\langle R\rangle(\neg p \vee q)$. A modal formula $A$ is positive in $p$ (negative in $p$ ) if all occurrences of $p$ in $A$ are positive (negative). A modal formula is called positive (negative) if it is positive (negative) in all proposition letters occurring in it.

A boxed atom is a formula of the form $\left[R_{i_{1}}\right] \ldots\left[R_{i_{n}}\right] p(n \geq 0)$ where $\left[R_{i_{1}}\right], \ldots,\left[R_{i_{n}}\right]$ are boxes of the language. If $n=0$ the boxed atom is the proposition letter $p$.

A Sahlqvist antecedent is a formula built up from $\perp, \top$, boxed atoms, and negative formulas using $\wedge$, $\vee$ and existence modal operators $(\langle R\rangle)$. A Sahlqvist implication is an implication $A \rightarrow B$ in which $B$ is positive and $A$ is a Sahlqvist antecedent.

A Sahlqvist formula that is build up from Sahlqvist implications by freely applying boxes and conjunctions, and by applying disjunctions only between formulas that do not share any proposition letters. An example of Sahlqvist formulas is $\left[R_{1}\right]\left(p \rightarrow\left\langle R_{2}\right\rangle p\right)$. Typically forbidden combinations in Sahlqvist antecedent are "boxes over diamonds", as in $\left[R_{1}\right]\left\langle R_{2}\right\rangle p \rightarrow\left\langle R_{3}\right\rangle\left[R_{4}\right] p$.

We know that every Sahlqvist formula is canonical for the first-order property it defines [6]. This firstorder property is computable from Sahlqvist formula by the Standard Translation. For example let us check this for MISG9:

$$
\begin{aligned}
& \left.p \wedge q \rightarrow\left\langle\mathrm{on}_{2}^{-1}\right\rangle\left(\left\langle\equiv_{1}\right\rangle\left(\left\langle\mathrm{on}_{2}\right\rangle p \wedge\left\langle\mathrm{on}_{2}\right\rangle q\right) \wedge\left\langle\mathrm{on}_{2}\right\rangle p\right)\right) \\
& \mathbb{1} \\
& S T_{z}\left(\left\langle\mathrm{on}_{2}^{-1}\right\rangle\left\langle\mathrm{on}_{2}\right\rangle p \wedge q\right) \rightarrow S T_{z}\left(\left\langle\mathrm{on}_{2}^{-1}\right\rangle\left(\left\langle\equiv_{1}\right\rangle\left(\left\langle\mathrm{on}_{2}\right\rangle p \wedge\left\langle\mathrm{on}_{2}\right\rangle q\right) \wedge\left\langle\mathrm{on}_{2}\right\rangle p\right)\right) \\
& \mathbb{1} \\
& (\forall P, Q)\left(( \exists x ) ( z \mathrm { on } _ { 2 } ^ { - 1 } x \wedge ( \exists t ) ( x \mathrm { on } _ { 2 } t \wedge P t ) \wedge Q z ) \rightarrow ( \exists y ) \left(z \mathrm{on}_{2}^{-1} y \wedge\right.\right. \\
& \left((\exists v)\left(y \not_{1} v \wedge\left((\exists w)\left(v \text { on }_{2} w \wedge P w\right)\right) \wedge(\exists h)\left(v \text { on }_{2} h \wedge Q h\right)\right) \wedge(\exists s)\left(y \mathrm{on}_{2} s \wedge P s\right)\right) \\
& \mathbb{1} \\
& (\forall P, Q)(\forall x, t)\left(z \mathrm { on } _ { 2 } ^ { - 1 } x \wedge x \mathrm { on } _ { 2 } t \wedge P t \wedge Q z \rightarrow ( \exists y ) \left(z \mathrm{on}_{2}^{-1} y \wedge\right.\right. \\
& \left((\exists v)\left(y \not \equiv_{1} v \wedge\left((\exists w)\left(v \text { on }_{2} w \wedge P w\right)\right) \wedge(\exists h)\left(v \text { on }_{2} h \wedge Q h\right)\right) \wedge(\exists s)\left(y \mathrm{on}_{2} s \wedge P s\right)\right)
\end{aligned}
$$

we define

$$
\begin{aligned}
& \sigma(P)=\lambda u \cdot(u=t), \\
& \sigma(Q)=\lambda u .(u=z) .
\end{aligned}
$$

Note that $\sigma(P)$ and $\sigma(Q)$ are the minimal instantiations making the antecedent true, and we have:

$$
\begin{aligned}
& (\forall x, t)\left(z \mathrm{on}_{2}^{-1} x \wedge x \mathrm{on}_{2} t \wedge t=t \wedge z=z \rightarrow(\exists y)\left(z \mathrm{on}_{2}^{-1} y \wedge\right.\right. \\
& \left((\exists v)\left(y \not_{1} v \wedge\left((\exists w)\left(v \text { on }_{2} w \wedge w=t\right)\right) \wedge(\exists h)\left(v \text { on }_{2} h \wedge h=z\right)\right) \wedge(\exists s)\left(y \text { on }_{2} s \wedge s=t\right)\right) \\
& \mathbb{1} \\
& (\forall x, t)\left(z \mathrm{on}_{2}^{-1} x \wedge x \mathrm{on}_{2} t \rightarrow(\exists y, v)\left(z \mathrm{on}_{2}^{-1} y \wedge y \not \equiv_{1} v \wedge v \mathrm{on}_{2} t \wedge v \mathrm{on}_{2} z \wedge y \mathrm{on}_{2} t\right)\right. \\
& \Uparrow \\
& (\forall x, t)(\exists y, v)\left(x \mathrm{on}_{2} z \wedge x \mathrm{on}_{2} t \rightarrow y \mathrm{on}_{2} z \wedge y \not z_{1} v \wedge v \mathrm{on}_{2} t \wedge v \mathrm{on}_{2} z \wedge y \mathrm{on}_{2} t\right)
\end{aligned}
$$

this is a local correspondent, then the modal formula also has a first-order global correspondent:

$$
(\forall x, z, t)(\exists y, v)\left(x \mathrm{on}_{2} z \wedge x \mathrm{on}_{2} t \rightarrow y \mathrm{on}_{2} z \wedge y \not_{1} v \wedge v \mathrm{on}_{2} t \wedge v \mathrm{on}_{2} z \wedge y \mathrm{on}_{2} t\right)
$$


this is the axiom I9. Note that MISG* and MISG ${ }^{(* *)}$ define the quasi-standard property [15]. In addition, the inference rules (MP), (NR) and (SR) are well-known in a normal modal system. The rule (IR) characterizes the irreflexivity of relation $\neq$.

\section{Completeness of MISG}

Since we had built an axiomatic system whose axioms are Sahlqvist formulae, to prove the completeness of MISG we will use the Venema's SD-theorem [17], which implies completeness of a wide variety of polymodal logics containing difference modality $[\neq]$, the irreflexivity rule (IR) for $[\neq]$ and satisfying some general conditions:

1. each modal operator $[R]$ must contain the "converse" operator $[R]^{-1}$ connected with $[R]$ by the following axioms from tense logic:

$$
\langle R\rangle\left[R^{-1}\right] A \rightarrow A, \quad\left\langle R^{-1}\right\rangle[R] \rightarrow A,
$$

2. the logic contains the axioms $\neq$-symmetry, $\neq$-pseudo-transitivity and $\neq$-inclusion,

3. all additional axioms should be Sahlqvist form.

Then the SD-theorem states that the logic is complete in the class of frames modally definable by the additional axioms.

Theorem 3 (The main theorem. Completeness.) A modal formula is a theorem of MISG if and only if it is valid in all incidence spatial frames.

PROOF. It is easy to see that additional axioms of MISG are in a Sahlqvist form, and by the SD-theorem, MISG is complete in the class of all incidence spatial frames.

\section{About other geometries}

We extend our results to other well-know spatial geometries: projective, affine and hyperbolic.

\subsection{The modal logic of projective spatial geometry}

In this subsection we present a modal logic of spatial projective geometry denoted by MSPG. The language of MSPG is the same as MISG. Semantically we can define MSPG as the logic of the class of all projective spatial frames.

Theorem 4 The class of spatial projective frames is modally definable in the class of all incidence spatial frames by the addition of the following formulas:

MPSG1 $\left\langle\mathrm{on}_{3}\right\rangle\left\langle\mathrm{on}_{3}^{-1}\right\rangle p \rightarrow\left\langle\mathrm{on}_{1}^{-1}\right\rangle\left\langle\mathrm{on}_{1}\right\rangle p$,

MPSG2 $\left\langle\mathrm{on}_{1}\right\rangle\left(p \wedge\left\langle\mathrm{on}_{1}^{-1}\right\rangle q\right) \rightarrow\left\langle\not_{1}\right\rangle\left(\left\langle\mathrm{on}_{1}\right\rangle p \wedge\left\langle\not_{1}\right\rangle q\right)$.

PROOF. The formulas MPSG1 and MPGS2 corresponds to the axioms SPF1 and SPF2 of spatial projective frames.

The formulas MPSG1 and MPGS2 are in Sahlqvist form, consequently the completeness is given by SD-theorem. 


\subsection{The modal logic of spatial affine geometry}

We introduce a modal logic of spatial affine geometry MSAG by extension of MISG. The language of MSAG contains a new modal operator denoted by [II]. The semantics of MSAG is in the class of all afine frames and the operator $[\|]$ is interpreted by the relation $\|$. Semantically, we can define MSAG as the logic of all affine frames.

Theorem 5 The class of all spatial affine frames is modally definable in the class of incidence spatial frames by addition of the following formulas:

Parallel $\left\langle\mathrm{on}_{3}\right\rangle\left\langle\mathrm{on}_{3}^{-1}\right\rangle p \rightarrow\langle\|\rangle p \vee\left\langle\mathrm{on}_{1}^{-1}\right\rangle\left\langle\mathrm{on}_{1}\right\rangle p$,

$$
\begin{aligned}
& \left\langle\equiv_{2}\right\rangle p \rightarrow\langle\|| p, \\
& {\left[\equiv_{2}\right] q \wedge\left\langle\mathrm{on}_{1}^{-1}\right\rangle\left\langle\mathrm{on}_{1}\right\rangle([\neq] p \wedge \neg q) \rightarrow[\|] p,}
\end{aligned}
$$

MASG1 $\langle U\rangle p \rightarrow\left\langle\mathrm{on}_{1}\right\rangle\langle\|\rangle p$,

MASG2 $\langle\|\rangle p \rightarrow\langle\|\|\rangle\|\| p$.

The formulas are in Sahlqvist form, then by SD-theorem we have completeness.

\subsection{The modal logic of spatial hyperbolic geometry}

We introduce a modal logic of hyperbolic spatial geometry MHSG by extension of MISG. The language of MHSG is the same that MASG. Semantically, we can define MHSG as the logic of all hyperbolic frames.

Theorem 6 The class of all hyperbolic frames is modally definable in the class of incidence spatial geometry by addition of the following formulas:

Parallel

MHSG1 $\langle U\rangle p \wedge q \rightarrow\left\langle\mathrm{on}_{1}\right\rangle\left(\langle\|\rangle p \wedge\left\langle\not_{2}\right\rangle\left(\langle\| \mid\rangle p \wedge\left\langle\mathrm{on}_{1}^{-1}\right\rangle q\right)\right) \vee\langle U\rangle\left(\left\langle\mathrm{on}_{1}\right\rangle p \wedge q\right)$.

In the same way, we have completeness.

\section{References}

[1] Balbiani, P. And Goranko, V., (2002). Modal Logics for Parallelism, Orthogonality and Affine Geometries, J. of Applied Nonclassical Logics, 12, 3-4, 365-397.

[2] BAlbiani, P., (2006). An expresive two-sorted spatial logic for plane projective geometry. Advances in Modal Logic, 49-68.

[3] Balbiani, P. and Callita. K., (2004). Solving constraints between lines in Euclidean Geometry. Lecture Notes in Computer Science 3192, 148-157.

[4] Balbiani, P. And Tinchev, T., (2004). Line-Based Affine Reasoning in Euclidean Geometry. Lecture Notes in Computer Science 3229, 474-486.

[5] VAn Benthem, (1983). The Logic of Time, Reidel.

[6] Blackburn, P., Rijke, M. And Venema, Y., (2001). Modal Logic, Cambridge University Press, United Kingdom.

[7] Coxeter, H., (1971). Fundamentos de Geometría, Limusa. 
[8] GabBay, D., (1981). An irreflexivity lemma with applications to axiomatizations of conditions on tense frames. In Aspects of Philosophical Logic, U. Mönnich, ed., pp. 67-89. Reidel.

[9] Goldblatt, R., (1987). Logics of Time and Computation. Lecture Notes Number 7. Center for the Study of Language and Information.

[10] Goldblatt, R., (1979). Topoï, the Categorial Analysis of Logic. North-Holland, Amsterdam, Netherlands.

[11] Greenberg, Marvin J., (1993). Euclidean and non-Euclidean Geometries, Freeman and Company, USA.

[12] Hilbert, D., (1971). Les Fondements de la Géométrie, Dynod, Paris.

[13] Jacobson, N., (1980). Basic Algebra II, W. H. Freeman and Company, New York.

[14] Ríder, A. And Rubio, R., (2004). Una lógica modal para la geometría plana de Lobachevski, Rev. Acad. Colom. Cienc., 28 (106), 87-94.

[15] Ríder, A. AND Rubio, R., (2005). A Modal Logic for Incidence Spherical Geometry, RACSAM, 99 (1), 79-117.

[16] Vakarelov, D., (1992). A modal theory of arrow. Arrow logics I, In Proceedings of the logic in AI. European Workshop JELIA' 92, Berlin, Germany, September 1992. D. Pearce and G. Wagner eds., 1-24. Vol. 633 of Lectures Notes in Artificial Intelligence. Springer-Verlag, Berlin.

[17] Venema, Y., (1993). Derivation rules as anti-axioms in modal logic. Journal of symbolic Logic, 58, 1003-1034.

[18] Venema, Y. And other, (2001). Modal Logic, Cambridge University Press.

[19] Wright, G., (1979). A modal logic of space. In The Philosophy of Nicholas Rescher, F. Sosa, ed. Reidel, Dordrecht.

Alfonso Ríder Moyano

Departamento de Matemáticas

Universidad de Córdoba

malrimoa@uco.es
Rafael María Rubio Ruiz

Departamento de Matemáticas Universidad de Córdoba

ma1rururauco.es 